\title{
Hyperinflammation bei SARS-CoV-2-Infektion
}

Zu Beginn der Pandemie deutete alles darauf hin, dass bei Kindern keine schweren Verläufe bei SARS-CoV-2-Infektionen auftreten. Doch seit Ende April 2020 häufen sich die Berichte von Kindern mit schweren Entzündungsreaktionen.

Die Pathophysiologie dieser Erkrankung ist noch nicht geklärt; ein Zusammenhang mit COVID-19 ergibt sich aus der Beobachtung, dass der Erkrankungsgipfel mit etwa vier Wochen Verzögerung dem von SARS-CoV-2 (Gesamtbevölkerung) folgt und einige der Kinder positiv getestet wurden.

Da es sich um ein neues Krankheitsbild handelt, hat man sich noch nicht auf eine einheitliche Namensgebung geeinigt. So bezeichnet das Europäische Zentrum für Prävention und Kontrolle von Krankheiten (ECDC) das Syndrom als PIMS-TS für "Paediatric inflammatory multisystem syndrome temporally associated with SARS-CoV-2". Die American Academy of Pediatrics und die WHO sprechen dagegen von MIS-C' für "Multisystem Inflammatory Syndrome in Children“. Letztere schlagen folgende Diagnoserichtlinien für MIS-C vor:

- Alter <21 Jahre mit Fieber

- Laborbefunde mit Beweis von Inflammation

Quelle: Dr. Marion Hofmann-Aßmus, SpringerMedizin.de (basierend auf: Hedrich C. Hyperinflammation bei SARSCoV-2-Infektionen

- Konzepte und Behandlung. Kinderund Jugendmedizin aktuell - Online-

Update 2020,

18.09.2020, virtuell)
„Das klinische Bild gleicht häufig einem Kawasaki Syndrom (KS), etwa zwei Drittel unserer Patienten erfüllten die klassischen oder inkompletten Kawasaki-Kriterien", erklärte Prof. Christian Hedrich, der an der University of Liverpool 29 betroffene Patienten untersuchte.

\section{Gastrointestinale Symptome bis hin zum akuten Abdomen}

Zusätzlich zu den Kawasaki-Kriterien traten hier zahlreiche weitere Symptome auf, etwa respiratorische und neurologische Anzeichen, sowie sehr häufig gastrointestinale Symptome bis hin zum akuten Abdomen.

》) Etwa die Hälfte der Betroffenen war über fünf Jahre alt

Als nicht KS-typisch fiel das Alter der Patienten auf, etwa die Hälfte der Betroffenen war über fünf Jahre alt. Einige Auffälligkeiten des Blutbildes passten ebenfalls nicht zur KS-Diagnose, beispielsweise Lymphopenie (79,3\%), Thrombopenie $(34,4 \%)$ oder Hypertriglyzeridämie (63\%).

\section{Immunglobuline überhaupt erforderlich?}

Die Therapie erfolgt derzeit rein empirisch und erfordert eine intensivmedizinische Überwachung. Wie Hedrich berichtete, wurden die Patienten in Liverpool größtenteils mit einer (39,3\%) bzw. zwei Dosen (60,7\%) intravenösen Immunglobulinen (IVIG) behandelt.

Sechs Patienten waren nach der ersten Dosis IVIG afebril, ein Patient bekam zusätzlich oral Kortikosteroide nach IVIG. Eine Patientin mit Verdacht auf Hyperinflammation nach einer vermuteten SARS-CoV-2-Infektion wurde erfolgreich mit hoch-dosiertem Anakinra behandelt und konnte nach acht Tagen aus der Klinik entlassen werden. Die übrigen Patienten (23/28) erhielten IVIG kombiniert mit intravenösem Methylprednisolon (IVMP) (9/28) oder zunächst IVIG alleine (2/28) und bei fehlendem Ansprechen IVMP alleine (2/28) oder IVIG plus IVMP (11/28).

„Unter IVMP plus IVIG entfieberten die Patienten schneller als mit IVIG alleine. Das stellt uns vor die Frage, ob Immunglobuline überhaupt erforderlich sind", konstatierte Hedrich. Dies könnten nur weitere Daten bzw. klinische Studien klären.

Hinweis des Verlags. Der Verlag bleibt in Hinblick auf geografische Zuordnungen und Gebietsbezeichnungen in veröffentlichten Karten und Institutsadressen neutral.

rheuma plus $2020 \cdot 19: 202$ https://doi.org/10.1007/s12688020-00385-9

๑) Springer-Verlag GmbH Austria, ein Teil von Springer Nature 2020 